\title{
Analytical evaluation of the lifetime of a three- axle bogie frame using the analytical model validated through experimental modal analysis
}

\author{
Manea Ion $^{1 *}$, Sebesan Ioan $^{2}$, Matache Mihai ${ }^{3}$, Prenta Gabriel $^{1}$ and Firicel Catalin ${ }^{1}$ \\ ${ }^{1}$ SC Softronic SRL, Calibration and Mechanical Testing Laboratory, Craiova, No.40, Dolj, Romania \\ ${ }^{2}$ Polytechnic University of Bucharest, Sector 6, Splaiul Independentei, No. 313, Romania \\ ${ }^{3}$ INMA Bucharest, Sector 1, Blv. Ion Ionescu de la Brad, No. 6, Romania
}

\begin{abstract}
The fatigue strength validation by tests of the railway bogie frames requires existence of an expensive laboratory and a long time for testing, of about 6 to 10 months. Considering these aspects, the european norms admit that fatigue tests can be replaced by finite elementes analysis, with condition that analytical model to be correctly realized and validated by tests. Experimental modal analysis provides a powerful tool for validation of the FEA model through experimental data. The article presents an application for analytical assessment of the fatigue strength for a three-axle locomotive bogie frame, before sending it to the laboratory fatigue tests. Using Ansys, it was carried out the structural analysis of the bogie frame, rezulting the modal model characterized by the modal parameters: eigenfrequencies and eigenshapes. The analytical model was validated through an EMA application carried out on the bogie frame and correlation analysis of the EMA and FEA models. The analytical model and data files containing the simulated vertical, transverse and twist loads, applied to the bogie frame according to EN13749/2011, constitutes the input data for nCode program, that evaluates the bogie frame lifetime using appropriate stress curves and a recognized hypothesis of damage accumulation.
\end{abstract}

\section{Background of EMA and FEA analysis}

Experimental Modal Analysis (EMA) is the procedure of building the mathematical model of a structure based on the experimental data obtained by measurements made on the structure brought into a controlled vibrational state. The system is excited under well-defined conditions and, determining the evolution laws of excitation and vibratory response, is identified the modal model characterized by a minimum number of modal parameters, describing its vibration eigenmodes: eigenfrequencies, damping factors and eigenshapes.

The most general EMA mathematical model which describes the dynamics of a system with $\mathrm{N}$ degrees of freedom (DOF) is formulated as a system of differential equations adapted to the calculation method, which can be in time domain or in frequency domain. Equation (1) shows the general form of the system moving equations for the time domain, and equation (2) shows the general form of the moving equations for the frequency domain [1].

\footnotetext{
*Corresponding author: ion.manea@softronic.ro
} 


$$
\begin{gathered}
{[M]\{\ddot{x}(t)\}+[C] \cdot\{\dot{x}(t)\}+[K]\{x(t)\}=\{F(t)\}} \\
-\omega^{2}[M]\{X(\omega)\}+i \omega[C] \cdot\{X(\omega)\}+[K]\{X(\omega)\}=\{Q(\omega)\}
\end{gathered}
$$

The unknown matrices elements of mass, stiffness and damping must first be estimated from experimental data, through measurements carried out in time or frequency domains. Once the matrices elements were estimated, the modal parameters are found as a solution of an eigenvectors and eigenvalues problem. Corresponding to equations (1) and (2), the most mathematical models, for description of dynamic response, can be arranged in a form of unit impulse response functions, $h_{p q}(t)$, or frequency response functions, $H_{p q}(\omega)$, in such a way to be described by the following relations in the time domain (3) or frequency domain (4) :

$$
\begin{gathered}
h_{p q}(t)=\sum_{r=1}^{N} A_{p q r} e^{\lambda_{r} t}+A_{p q r}^{*} e^{\lambda_{r}^{*} t} \\
H_{p q}(\omega)=\sum_{r=1}^{N} \frac{A_{p q r}}{i \omega-\lambda_{r}}+\frac{A_{p q r}^{*}}{i \omega-\lambda_{r}^{*}}
\end{gathered}
$$

Unit impulse response functions are rarely or never directly measured, but they are calculated from associated frequency response functions (FRF) via the inverse FFT.

It have been used the following notations: $[\mathrm{M}],[\mathrm{C}],[\mathrm{K}]$, the mass, damping and stiffness matrices; $\{\ddot{x}(t)\},\{\dot{x}(t)\},\{x(t)\}$, the vectors of acceleration, velocity and displacement; $\{F(t)\}$ generalized vector of forces; $\omega$, angular frequency $(\mathrm{rad} / \mathrm{sec}) ; \mathrm{p}$, measured DOF of response; $\mathrm{q}$, measured DOF of input; $\mathrm{r}$, modal number vector; N, number of modal frequencies; $A_{p q r}$, residue for mode $\mathrm{r} ; \lambda_{r}$, system pole for mode $\mathrm{r}$.

In terms of Finite Element Analysis (FEA), the structure is divided into finite elements between which the mathematical connections are established, equivalent to the physical connections between the adjacent elements or the environment. To the physical model corresponds a mathematical model, similar to the mathematical model of EMA, difference being that in the FEA model are not considered the damping of system:

$$
[M] \cdot\{\ddot{x}\}+[K] \cdot\{x\}=\{0\}
$$

Solving the system of differential equations leads to the analytical determination of the eigenfrequencies and eigenshapes which, referring to the same system, must to be the same as those determined by experimental EMA. Finite element analysis works with an approximate mathematical model, realized by more or less gross approximations of both geometric and material characteristics. Even if the mathematical model is perfect, it will still provide an approximate analytical model, because it is well known that the characteristics of the material used in the modelling never coincide with the real characteristics of the structure. In addition, it is well known that any geometrical modelling of a product is accompanied by more or less gross execution errors, errors that cannot be translated into geometric model.

It follows, from the above, that a good and reliable analytical model can be obtained only through validation with the data provided by the real system through EMA tests.

\section{FEA and EMA analysis of a three-axle locomotive bogie frame}

Finite Element Analysis. It was been used the SolidWorks for geometrical modelling and Modal and Structural modules of Ansys 15 for modal and structural analysis of bogie frame..

Analysis was made in linear elastic regime, the bogie frame being modelled as follows:

- Tetrahedral elements type Solid 92 in the number of: 806471 elements and 1490919 nodes. - Linear rigid beam elements type Beam, for the structure between the primary suspension and axle, for axle, and for the forces transmission from the application points to the structure. - Spring elements for the primary and secondary suspensions.

The analytical modal analysis was performed in the frequency range of 10 to $100 \mathrm{~Hz}$. Modal shapes, are presented in the correlation analysis section in connection with the EMA. 
Experimental Modal Analysis. EMA tests were carried out in Softronic Craiova-Romania on a three-axle bogie frame designed for LEMA $6000 \mathrm{~kW}$ electric locomotive. The bogie frame has been redesigned for extended range of operating temperatures of $-40^{\circ} \mathrm{C}$ to $+40^{\circ} \mathrm{C}$. The main material used is steel S355NL. To perform the EMA tests, the bogie frame was placed on four helicoidally springs, each having elastic constant $\mathrm{k}=519 \mathrm{~N} / \mathrm{mm}$, in normal operating position, the bogie frame can be considered as a free system, without restricting any DOF.

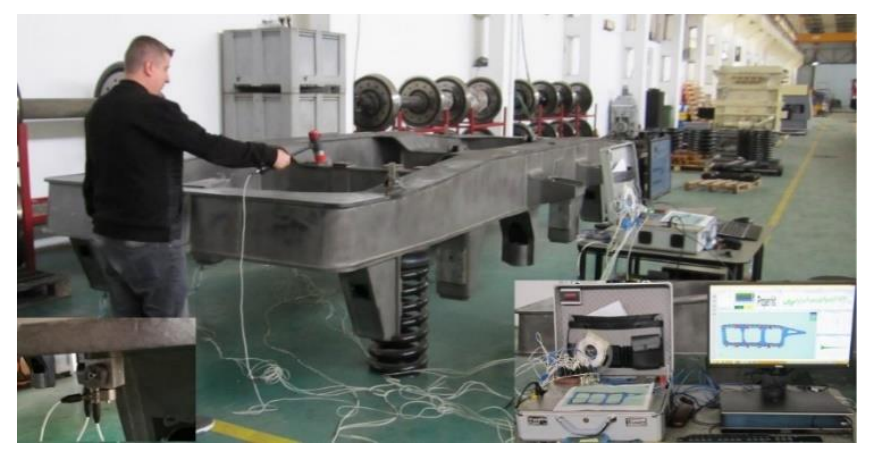

Fig. 1. Mounting of the three-axle locomotive bogie frame for EMA tests

The excitation of the bogie frame was made in only one point using the impact method with an impact hammer of $25 \mathrm{kN}$. It was used a mediation over 4 impacts to eliminate the noise in measurements and to obtain a high accuracy of FRF. The analysis was made in frequency range of 10 to $100 \mathrm{~Hz}$. The acceleration response was measured in a number of 45 points, for each measuring point the response being measured on three orthogonal directions: vertical, transverse and longitudinal, resulting a number of 135 DOF measured. The points of measurement have been selected to allow a suggestive animation of the structure.

For EMA tests was used the following excitation and measuring equipment and software: -Piezoelectric accelerometers type 355B03, $10.19 \mathrm{mV} /\left(\mathrm{m} / \mathrm{s}^{2}\right), \pm 490 \mathrm{~m} / \mathrm{s}^{2}, 15 \mathrm{pcs}$; -Impact Hammer type 086D20, $0.23 \mathrm{mV} / \mathrm{N}, \pm 22240 \mathrm{~N}$ pk, 1 pcs.;

-Softronic Data Acquisition System including: LAN-XI type 3053B120, 12 input chann., $25.6 \mathrm{kHz}$ and LAN-XI type 3160A042, 4 input chan., 2 output chan., $25.6 \mathrm{kHz}$;

-Structural Dynamics Test Consultants, a software working under PulseLabshop to provide a dedicated measurement environment for structural dynamic measurements;

-Pulse Reflex Modal Analysis, a post-processing module for classical modal analysis, using hammer or shaker excitation. It works with data measured as FRF and express the eigenmodes by modal parameters: damped frequency, damping, and residues;

-Pulse Reflex Correlation Analysis, provides a powerful tool to verify and validate the FEA modal model by true EMA modal model. The correlation analysis is accomplished by graphical comparisons, shape animations and numerical Modal Assurance Criterion (MAC).

Both modal models, FEA and EMA, were identified in the frequency range of 10 to $100 \mathrm{~Hz}$ and was performed the correlation analysis of the two models. Figure 2 presents the vector comparison panel with Cross Modal Assurance Criterion for the two models. Table 1 presents the results of FEA and EMA correlation analysis. There are presented the damped frequencies for the two modal models and the evaluation error of analytical damped frequency. It is presented the MAC criterion of modal shapes for the two models. Figures 3 to 7 presents the bogie frames in the first five eigenmodes. Analysing the presented data it can be concluded that:

-The damped frequency error estimated by FEA is less than 5\% compared to EMA;

-The MAC correlation criterion is greater than 0.6 ;

-Modal shapes calculated by FEA are very similar to modal shapes calculated by EMA. 


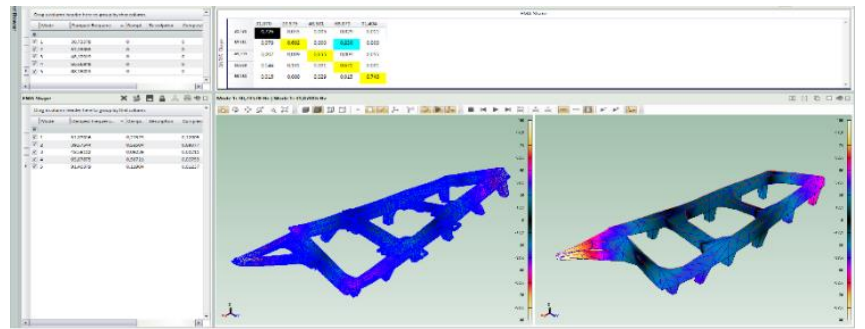

Fig. 2. Pulse Reflex Correlation - panel for Modal Assurance Criterion CrossMAC comparison

Table 1. Results of FEA - EMA correlation analysis

\begin{tabular}{|c|c|c|c|c|c|c|c|}
\hline \multirow{2}{*}{$\begin{array}{l}\text { Mod } \\
\text { No. }\end{array}$} & FEA & \multicolumn{3}{|c|}{ EMA } & \multirow{3}{*}{$\begin{array}{c}\text { Mode } \\
\text { direction }\end{array}$} & \multirow{2}{*}{$\begin{array}{l}\text { Frequency } \\
\text { Error }\end{array}$} & \multirow{3}{*}{\begin{tabular}{|c|} 
MAC \\
Modal \\
Assurance \\
Criterion
\end{tabular}} \\
\hline & $\begin{array}{c}\text { Damped } \\
\text { Frequency }\end{array}$ & $\begin{array}{c}\text { Damped } \\
\text { Frequency }\end{array}$ & Damping & Complexity & & & \\
\hline & $(\mathrm{Hz})$ & $(\mathrm{Hz})$ & $(\%)$ & & & $(\%)$ & \\
\hline 1 & 30.73 & 31.07 & 0.215 & 0.120 & Vertical & -1.106 & 0.726 \\
\hline 2 & 37.43 & 39.57 & 0.525 & 0.098 & Vertical & -5.717 & 0.602 \\
\hline 3 & 48.11 & 48.58 & 0.092 & 0.002 & Transverse & -0.998 & 0.655 \\
\hline 4 & 66.66 & 65.87 & 0.567 & 0.027 & Vertical & 1.185 & 0.671 \\
\hline 5 & 88.18 & 91.40 & 0.119 & 0.012 & Transverse & -3.652 & 0.746 \\
\hline
\end{tabular}

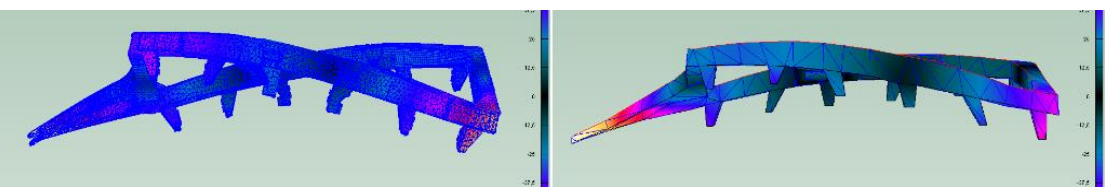

Fig. 3. The $1^{\text {st }}$ eigenmodes, $\mathrm{OZ}$ direction, left FEA $30.73 \mathrm{~Hz}$, right EMA $31.07 \mathrm{~Hz}$
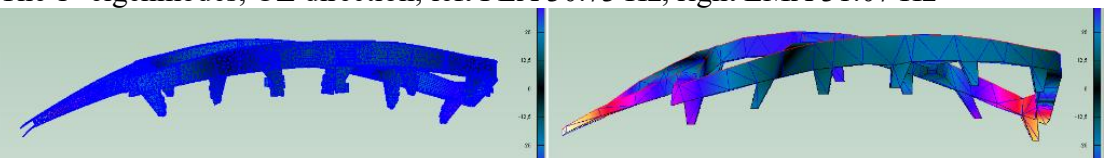

Fig. 4. The $2^{\text {nd }}$ eigenmodes, $\mathrm{OZ}$ direction, left FEA $37.43 \mathrm{~Hz}$, right EMA $39.57 \mathrm{~Hz}$
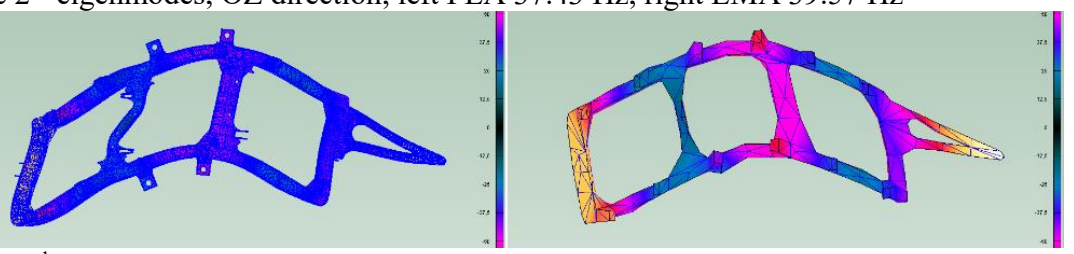

Fig. 5. The $3^{\text {rd }}$ eigenmodes, OY direction, left FEA $48.11 \mathrm{~Hz}$, right EMA $48.58 \mathrm{~Hz}$

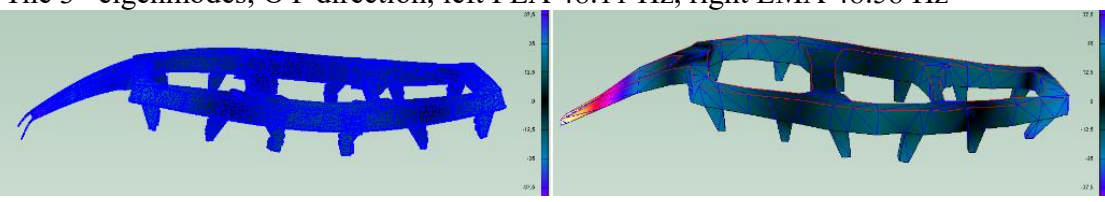

Fig. 6. The $4^{\text {th }}$ eigenmodes, $\mathrm{OZ}$ direction, left FEA $66.66 \mathrm{~Hz}$, right EMA $65.87 \mathrm{~Hz}$
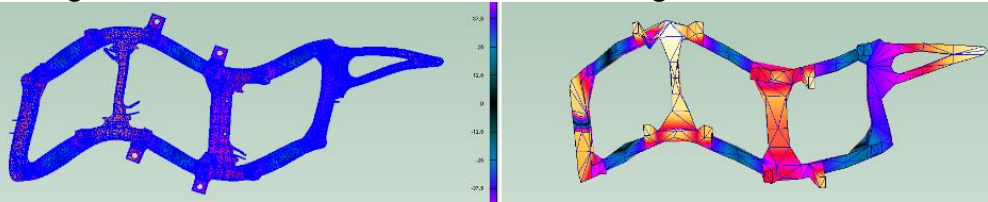

Fig. 7. The $5^{\text {th }}$ eigenmodes, Oy direction, left FEA $88.18 \mathrm{~Hz}$, right EMA $91.40 \mathrm{~Hz}$

According to table 1 and figures 3 to 7 , it can be concluded that the FEA model is a correctly calibrated model and can be used to evaluate the dynamic response of the bogie. 


\section{Analytical lifetime estimation of the bogie frame at service simulated loads}

The fatigue of a structure is caused by loading the structure with repetitive stresses for a time.

The traditional method to estimate the number of cycles of repetitive stresses, that a structure can withstand, use the empirical curves known as the S-N or Wholer curves. This technique is widely used today and is known as the "stress-life" approach. The lifetime estimation by stress-life approach uses the hypothesis that the stress remains in the elastic region, the method being suitable in fatigue analysis for large number of stress cycles, $\mathrm{N} \geq 10^{3}$.

If it is defined the time history of a load, with variable amplitude of stresses, several steps must be taken to calculate the lifetime from stresses applied to the individual cycles. In a first stage, the history of the load is decomposed into peak-valley sequences. Next, the reduced load sequence is processed with a standard cycle counting algorithm (like rainflow counting algorithm) and extracts individual stress cycles. Single stress cycle are then used together with S-N curves to calculate the lifetime associated with each stress cycle.

After a lifetime (or damage) is calculated for each individual stress cycle, it is necessary a hypothesis for determining the total lifetime, or cumulative damage. The most commonly hypothesis of damage assessment is based on the cumulative damage rule, or the PalmgrenMiner rule, which states that the total damage accumulated in the stress history is given by the linear sum of the partial damage generated by the individual cycle loads.

For the bogie frame, the fatigue laboratory tests are intended to confirm that the frame strength is sufficient with regard to the main loads acting on it. Loads taken into account in fatigue tests are those inducing stresses in the entire frame structure of the bogie frame, the main loads being: vertical forces, transverse forces and twist input. The tests consist in strain measurements in the highly stressed areas of the bogie frame. Generally, the fatigue tests are carried out in successive stages, the first stage corresponding to demonstrating that the bogie frame can withstand to service loads encountered on entire projected lifetime. The first stage consists of $6 \times 10^{6}$ cycles of application of the vertical and transverse forces, and $6 \times 10^{5}$ cycles of application of the twist loads. The table 2 presents the loads for LEMA bogie frame.

Table 2. Combination of loads for fatigue tests of LEMA locomotive three-axle bogie frame

\begin{tabular}{|c|c|c|}
\hline Type of load & Type of component & Load amplitude \\
\hline \multirow{2}{*}{ Fy, transversaly applied to each axle } & quasi-static part & $26476 \mathrm{~N}$ \\
\cline { 2 - 3 } & dynamic part & $26476 \mathrm{~N}$ \\
\hline \multirow{2}{*}{$\begin{array}{c}\text { Fz, vertical, applied in the middle on } \\
\text { each sideframe }\end{array}$} & static part & $174579 \mathrm{~N}$ \\
\cline { 2 - 3 } & quasi-static part & $17458 \mathrm{~N}$ \\
\cline { 2 - 3 } & dynamic part & $34916 \mathrm{~N}$ \\
\hline Twist, vertical applied to a sideframe & quasi-static part & $0.5 \%$ \\
\hline
\end{tabular}

In order to eliminate possible failure of bogie frame during laboratory tests, a preliminary theoretical analysis of the frame lifetime was performed on the loads mentioned in table 2 .

With the FEA model, validated by EMA experimental data, was run a static analysis of the bogie frame subject to action of static loads mentioned above. The results of stress distribution on the bogie frame were saved as file with .rst extension.

Was simulated a cycle with experimental data mentioned in table 2. The simulated data file, containing the loads for testing, and .rst file constitutes inputs data for the HBM Prenscia nCode 13.1 software, module DesignLife, which evaluates fatigue damage and lifetime on the basis of Wholer curves and Palmgren-Miner hypothesis of cumulative damage.

Figure 8 presents the nCode logical scheme to evaluate the lifetime of bogie frame under loads in the first step of fatigue laboratory tests, corresponding to the normal operating loads. In upper left, it is presented the time history of vertical forces, transverse forces and twist. 
The upper centre presents the results of running the Rainflow module that gives the statistical distribution of stress cycles related to average value and the stress level. In the upper right, are shown the stress distribution on the bogie frame, for the original static load and for the dynamic loading with loads mentioned in table 2. The lower table shows the numerical results of analysis, with distribution of main parameters on nodes and elements. The lifetime of each node/element is presented. It can be seen from the table that for the most stressed areas of the frame, the lifetime is of $4.65 \times 10^{7}$ lengths of record, or equivalent $9.30 \times 10^{8}$ dynamic cycles, much more than those $6 \times 10^{6}$ cycles imposed by EN 13749 for the first stage of fatigue tests.

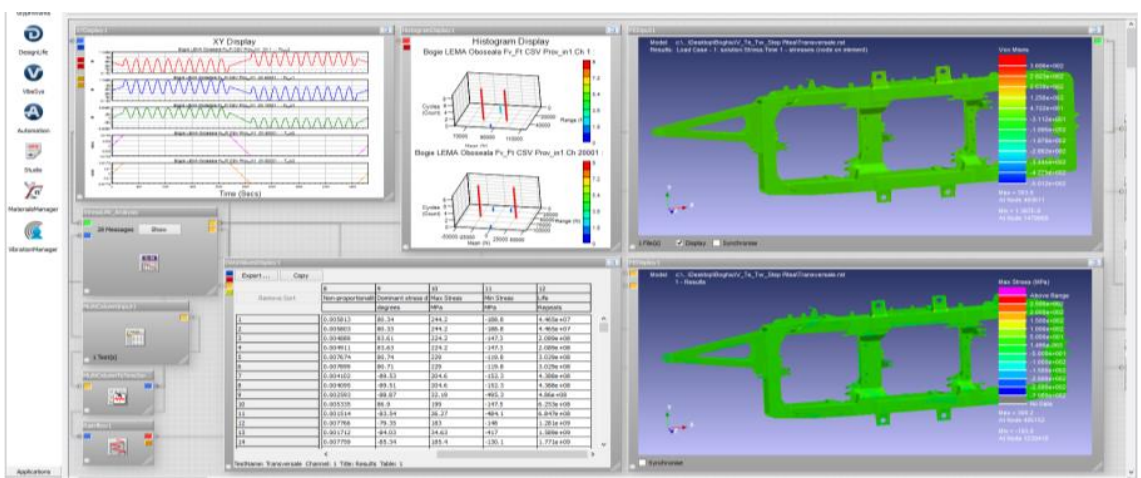

Fig. 8. Logical scheme to evaluate the service lifetime of the LEMA $6000 \mathrm{~kW}$ bogie frame

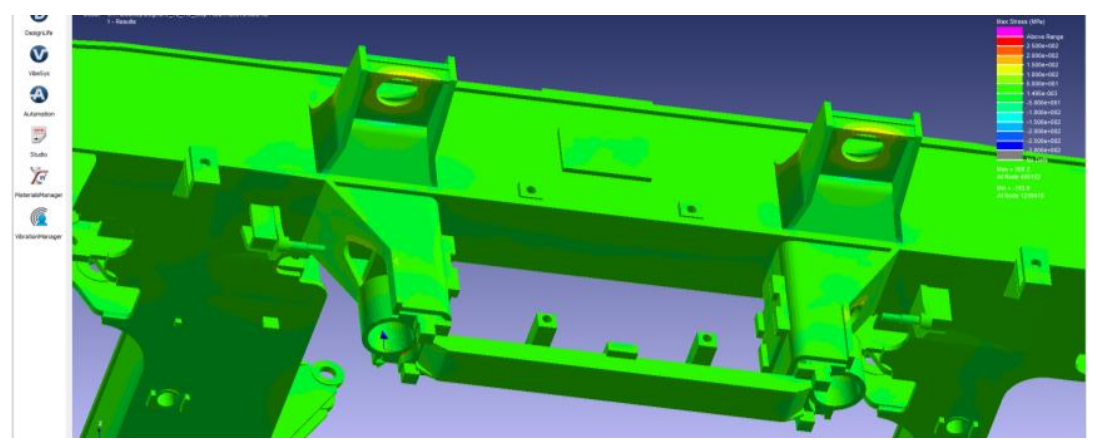

Fig. 9. Detail for stress distribution, in $\mathrm{MPa}$, in the most stressed areas of the bogie frame

\section{Conclusions}

The article presents an application for validating the FEA analytical model of a bogie frame through the real modal model realized by experimental modal analysis, EMA. The validated analytical model is used to assess the lifetime of the bogie frame, corresponding to the service loads to which the bogie frame will be subjected during the laboratory fatigue tests.

At the moment, the method outlined in the paper is under analysis and development.

This work was supported by a grant of the Romanian National Authority for Scientific Research and Innovation, CNCS-UEFISCDI, project number PN-III-P2-2.1-PTE-2016-0008, within PNCDI III

\section{References}

[1] D.J. Ewins, Modal Testing, Theory, Practice, John Wiley and Sons, Inc., New York 1984.

[2] EN 13749, Railway applications - Wheelsets and bogies - Method of specifying the structural requirements of bogie frames, March 2011. 